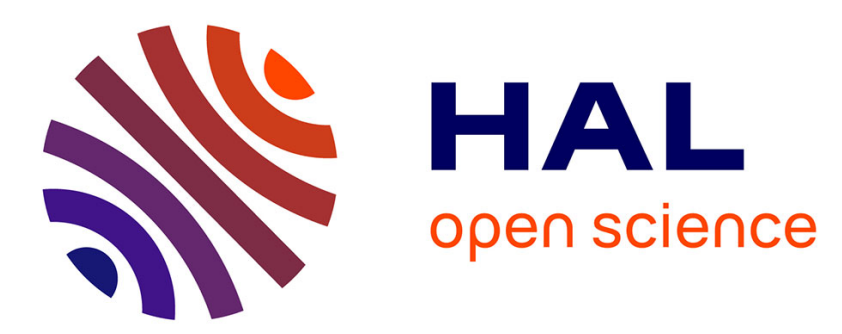

\title{
Characterization of circumferential guided waves in a cylindrical cortical bone-mimicking phantom
}

Pierre Nauleau, Etienne Cochard, Jean-Gabriel Minonzio, Quentin Grimal, Pascal Laugier, Claire Prada

\section{- To cite this version:}

Pierre Nauleau, Etienne Cochard, Jean-Gabriel Minonzio, Quentin Grimal, Pascal Laugier, et al.. Characterization of circumferential guided waves in a cylindrical cortical bone-mimicking phantom. Journal of the Acoustical Society of America, 2012, 131 (4), pp.EL289 - EL294. 10.1121/1.3687016 . hal-01394321

\section{HAL Id: hal-01394321 \\ https://hal.sorbonne-universite.fr/hal-01394321}

Submitted on 9 Nov 2016

HAL is a multi-disciplinary open access archive for the deposit and dissemination of scientific research documents, whether they are published or not. The documents may come from teaching and research institutions in France or abroad, or from public or private research centers.
L'archive ouverte pluridisciplinaire HAL, est destinée au dépôt et à la diffusion de documents scientifiques de niveau recherche, publiés ou non, émanant des établissements d'enseignement et de recherche français ou étrangers, des laboratoires publics ou privés. 


\title{
Characterization of circumferential guided waves in a cylindrical cortical bone-mimicking phantom
}

\author{
Pierre Nauleau ${ }^{\text {a) }}$ \\ Laboratoire d'imagerie paramétrique, UPMC Univ Paris 06, CNRS UMR 7623, \\ 15 rue de l'école de médecine, F-75006 Paris, France
}

\begin{abstract}
Etienne Cochard
Institut Langevin, ESPCI ParisTech, Université Denis Diderot-Paris7, CNRS UMR 7587, 10 rue Vauquelin, F-75005, Paris, France

Jean-Gabriel Minonzio, Quentin Grimal, Pascal Laugier Laboratoire d'imagerie paramétrique, UPMC Univ Paris 06, CNRS UMR 7623, 15 rue de l'école de médecine, F-75006 Paris, France

Claire Prada

Institut Langevin, ESPCI ParisTech, Université Denis Diderot-Paris7, CNRS UMR 7587, 10 rue Vauquelin, F-75005 Paris, France
\end{abstract}

Date: December $9^{\text {th }}, 2011$

Running title: Characterization of a bone-mimicking phantom

a) Author to whom correspondence should be addressed. Electronic mail : pierre.nauleau@upmc.fr 


\begin{abstract}
The cortical shell of the femoral neck was recently demonstrated to act like a waveguide for circumferential waves. Femoral neck assessment with ultrasound could be enhanced by the measurement of guided waves. In this study, the DORT method is used to measure the phase velocities of circumferential guided modes in a tube of circular cross-section with dimensions characteristic of femoral neck. The tube is made of a bone-mimicking material. Five guided modes are obtained and compared to theoretical predictions. The work substantiates the feasibility of measuring guided waves in a relatively thick tube of attenuating material with the DORT method.

(C) 2011 Acoustical Society of America
\end{abstract}

PACS numbers: 43.80.Vj, 43.80.Ev, 43.20.Mv 


\section{Introduction}

Osteoporotic fractures are one of the most common causes of disability and an important part of medical care costs. ${ }^{1}$ Among osteoporosis related fractures, hip fractures are associated with the highest morbidity and mortality. ${ }^{1}$ The gold standard for fracture risk prediction is assessment of bone mineral density (BMD) using X-ray absorptiometry (DXA). Quantitative ultrasound techniques represent an alternative modality with capacities to quantitatively assess the bone status beyond BMD. The risk of hip fracture is best predicted by site-matched DXA measurements directly at the proximal femur. ${ }^{2}$ Furthermore, recent laboratory studies indicate that the cortical shell of the femoral neck plays an important role for fracture prevention. ${ }^{3-4}$ This motivates the focus of our study on measurements on cortical bone at the femoral neck. Our group has reported that the cortical shell acts like a waveguide supporting the propagation of circumferential waves. ${ }^{5}$ We hypothesize that measuring several guided wave modes at the femoral neck could enhance the assessment of its strength compared to our previously reported method based on the measurement of the time-of-flight of the first arriving signal. ${ }^{5}$ In this work, we investigate the feasibility of measuring the phase velocities of circumferential waves in a cortical bone mimicking phantom. The effectiveness of the DORT (Decomposition of the time reversal operator) method introduced by Prada et al. ${ }^{6}$ to measure phase velocities of circumferential waves was demonstrated for a thin hollow steel cylinder immersed in water. The question arises whether the DORT method can be applied to the femoral neck which characteristics are highly different of a thin steel tube: lower acoustical contrast for bone $(Z=7$ 9 MRayl $)^{7}$ than for steel $(Z=45$ MRayl) compared to water; higher attenuation in cortical bone $\left(2-5 \mathrm{~dB} . \mathrm{cm}^{-1}\right)^{8}$ compared to steel $\left(0.06 \mathrm{~dB} \cdot \mathrm{cm}^{-1}\right)$; higher thickness-to-wavelength ratio $(0.75)$ at 
$1 \mathrm{MHz}$ (frequency used for in vivo) for a typical femoral neck thickness compared to that in the thin steel shell (0.36).

In this letter we present the results of the first application of the DORT method to measure a hollow cylinder of circular cross-section made of a cortical bone-mimicking material. The objective was to assess the possibility to retrieve the phase velocities of several circumferential guided waves modes with a good precision.

\section{Rationale for the measurement of circumferential waves phase velocities with the DORT method}

A hollow tube, placed in a water tank, is excited by an incident wave. At a particular incidence angle $\theta$, a circumferential wave characterized by its phase velocity $c_{\phi}$, is generated. These two parameters are related to the velocity in water $c_{0}$, by the Snell-Descartes' law. This circumferential wave propagates in the shell and radiates in the external medium with the angle $\theta$. In the case of excitation by a plane wave, two circumferential waves are generated at symmetrical points A and B, Fig. 1. Trigonometry relates the distance between A and B, $d_{\mathrm{AB}}$, to the tube diameter $D$, and the angle $\theta$. Therefore, the phase velocity is directly related to $d_{\mathrm{AB}}$ as

$$
c_{\Phi}=c_{0} \frac{D}{d_{\mathrm{AB}}}
$$

The radiated waves are observed with an array in the $x$ direction. They seem to come from two virtual sources A' and B' aligned horizontally with A and B, but placed on the vertical diameter. Hence, the key point lies in locating the virtual sources for several modes, at several frequencies.

The time reversal principle can be used to focus on the virtual sources. However, if the received waves are mixed in time domain, different modes can not be distinguished. To 
overcome this limitation, a signal processing, called the DORT method, based on the matrix formalism of the time reversal process was proposed. ${ }^{6}$ At each frequency, the transfer matrix is decomposed using the singular value decomposition (SVD). Each singular vector is associated with a source; so the backpropagation of a singular vector yields focusing on a distinct source. Besides mode selection, a second interest of the method is the possible denoising: low energy singular vectors, related to noise, can be eliminated.

\section{Materials and methods}

The experiments were conducted on a hollow cylinder made of a cortical bone-mimicking material (Sawbones, Pacific Research Laboratory Inc., Vashon, WA). This material, consisting of short glass fibers embedded in epoxy resin, is transversely isotropic. The bulk velocities and attenuation coefficients of this composite, in the isotropy plane, were independently measured ${ }^{9}$ : $c_{\mathrm{L}}=2870 \pm 40 \mathrm{~m} \cdot \mathrm{s}^{-1}, \quad c_{\mathrm{T}}=1520 \pm 20 \mathrm{~m} \cdot \mathrm{s}^{-1}, \quad \alpha_{\mathrm{L}}=2.63 \pm 0.30 \mathrm{~dB} \cdot \mathrm{cm}^{-1} \cdot \mathrm{MHz}^{-1}$, $\alpha_{\mathrm{T}}=8.40 \pm 0.57 \mathrm{~dB} \cdot \mathrm{cm}^{-1} \cdot \mathrm{MHz}^{-1}$, where subscripts $\mathrm{L}$ and $\mathrm{T}$ stand for compression and shear waves. Attenuations are obtained in $\mathrm{dB} \cdot \mathrm{cm}^{-1} \cdot \mathrm{MHz}^{-1}$ from $(\alpha \lambda)$ in $\mathrm{Np}$ from Minonzio et al. ${ }^{9}$ and the following conversion coefficient $8.69 \times 10^{4} \times(\alpha \lambda)_{\mathrm{L}, \mathrm{T}} / c_{\mathrm{L}, \mathrm{T}}$. Mass density is $1.64 \mathrm{~g} . \mathrm{cm}^{-3}$. These values are representative of the mean acoustic properties generally reported for human cortical bone. $^{7-8}$ The structure of the femoral neck is rather complex; the shape and mean diameter of the cross-section vary along the neck axis. However, the medial half has a more circular shape. Then, for the purpose of the study, a hollow cylindrical tube of cortical bone-mimicking material represents a reasonable approximation of real neck in its mid part. The diameter and wall thickness of the tube were $26 \mathrm{~mm}$ and $2.1 \mathrm{~mm}$, respectively. These values correspond to the 
typical geometrical properties for the femoral neck. ${ }^{10}$ During the experiment, the phantom was immersed in a water tank and the inner cavity was filled with air.

The measurement of the transfer matrix of the system was performed with a 1-D array of transducers specifically designed in anticipation of future clinical in vivo measurements. The probe (Imasonic, Besançon, France) which is a cylindrically focused 128-element array was used for both emission and reception. The focal length was $160 \mathrm{~mm}$, the $-6 \mathrm{~dB}$ beam width was $1.7 \mathrm{~mm}$, the centre frequency $1 \mathrm{MHz}$ and the $-6 \mathrm{~dB}$ bandwidth was $0.6 \mathrm{MHz}$. In order to maximize the signal-to-noise ratio, the characteristics of the emission were chosen carefully: 128 orthogonal (Hadamard-Walsh basis) chirps were emitted. Each of them covered a frequency bandwidth of [0.4-1.6] MHz in $20 \mu \mathrm{s}$. A multi-channel electronic device (Lecoeur Electronique, Chuelles, France) was used to manage the 128 elements. Signals reflected after each excitation were recorded during $100 \mu$ s by all of the 128 elements, digitized at a rate of $20 \mathrm{MHz}$ and stored on a computer for off-line analysis.

The DORT method was implemented in a MATLAB program (The Mathworks Inc., Natick, MA). First, the signals corresponding to the radiated circumferential waves were time windowed. They were used to calculate the SVD of the transfer matrix at each frequency. Then, the user heuristically decided which singular vectors to keep. The selected singular vectors were numerically backpropagated to locate the virtual sources corresponding to the modes of the tube. Finally, the phase velocity and the wavenumber of each mode were calculated as a function of the frequency in the bandwidth [0.4-1.6] MHz, according to Eq. (1).

In the experiment, there can be several sources of bias like a misalignment of the tube or spurious reverberations on the tank wall. To evaluate the potential of the method in ideal conditions, a simulated experiment was performed. The simulation, based on the normal modes 
expansion, ${ }^{11}$ consists of calculating the signals scattered by the cylinder and recorded by the array. The phantom and probe properties were both taken into account in the simulation. The DORT method was then applied to the calculated received signals as for the experimental data.

The observed modes were identified by comparison with theoretical predictions from a plate model. The dispersion curves of a bone-mimicking plate loaded with water on one side and air on the other side were calculated using Disperse software (Imperial College, London, UK) fed with the material properties of the phantom, given above. The modes are named in accordance with those of a semi-immersed plate. ${ }^{12}$ In particular, the mode denoted A is added compared to the classical free plate case.

\section{Results and discussion}

Experimental signals received by the 128 transducers after one transmission are presented Fig. 2. Two main components can be distinguished. A first echo arriving approximately at $200 \mu$ s is associated with the specular reflection on the bone-mimicking phantom interface. Echoes arriving after $220 \mu$ s are associated with circumferential waves propagating in the tube wall. The magnitude of these signals, being approximately $-40 \mathrm{~dB}$ lower than the specular echo, is weak most likely as a result of both the impedance mismatch between water and the bone-mimicking material and the high attenuation of the material. Nevertheless, these signals corresponding to the circumferential waves are clearly visible. The experimental guided mode wavenumbers are presented with the results of the simulated experiment and the theoretical dispersion curves (semi-immersed plate model) in Fig. 3. 
Five branches of modes are observed in the frequency bandwidth [0.4-1.6] MHz. All the modes predicted by the simulation are experimentally observed. Thus it indicates the good quality of our experimental set-up. Moreover, there is an overall qualitative agreement with the theoretical predictions from the plate model. The branches observed experimentally can be associated with $A, A_{0}, A_{1}, S_{1}$ and $S_{2}$ plate modes. The branch corresponding to $A_{0}$ deviates from the plate prediction due to the influence of the curvature of the tube on this mode. In the seminal experiment, ${ }^{6}$ three modes were observed $\left(\mathrm{A}_{0}, \mathrm{~S}_{0}\right.$ and $\left.\mathrm{A}_{1}\right)$. In our configuration, more modes are obtained due to a larger frequency-thickness product. However, all the modes predicted by the plate model are not observed experimentally. For example, $\mathrm{S}_{2}$ is not measured for frequencies above $0.75 \mathrm{MHz}$ and only few data points are captured around the cut-off frequency of mode $\mathrm{A}_{3}$. Two reasons can explain this discrepancy. First, the proposed analysis is limited by the lateral resolution of our probe. In fact, the calculation of the wavenumbers is based upon the measurement of the distance between two virtual sources. When the distance between the sources is smaller than the lateral resolution, the associated wavenumbers can not be determined. This situation of two unresolved sources occurs for high velocities, i.e. low wavenumbers [Eq. (1)]. It explains why few points are obtained near the cut-off frequency for the mode $A_{3}$. The second reason deals with the mode fluid coupling: some modes may not be well excited because they are not coupled with the fluid, while other modes may be so well coupled that they decrease too fast before they reached the location of the virtual sources. This might be the case for the mode $\mathrm{S}_{2}$.

One limitation of this study arises from the use of a semi-immersed plate model. Although it appears to be a reasonable approximation to identify the modes experimentally 
observed, it can not account for the curvature effect of a tube on the propagation characteristics of some modes as, for example, $\mathrm{A}_{0}$.

Moreover, the measured cylindrical tube is only a rough approximation of the real femoral neck. First, the shape of the neck is neither circular nor regular and the cortical thickness may vary along the circumference. These issues were already identified and we are currently carrying out additional studies to extend the application of the DORT method to shells with a complex shape, for which a different relationship between the phase velocity and the location of the virtual sources [Eq. (1) for a cylinder] can be derived. Secondly, the modeling of the inner bone cavity, composed of trabecular bone filled with marrow, by an air-filled cavity is imperfect. In particular, the transmission through the inner cavity may generate echoes, reflected from the bottom wall, which may interfere with the circumferential waves. Here, we assumed that, since trabecular bone (the filling material of the cortical shell) is highly attenuating, ${ }^{13}$ the reflected signals should not prevent the observation of the circumferential waves. However, further studies are necessary to investigate this issue. Finally, the potential impact of surrounding soft tissues must be evaluated in view of future in vivo applications.

\section{Conclusion}

In this study, the DORT method was applied to measure the wavenumbers of circumferential guided modes in a cylindrical cortical bone-mimicking phantom. Several guided modes of the bone-mimicking phantom were identified and the obtained dispersion curves were in good agreement with theory. This suggests that the proposed method might be effective in 
characterizing the thickness and elastic properties of a waveguide close to bone. This initial step opens perspectives for the ultrasonic characterization of the femoral neck.

\section{Acknowledgments}

Authors would like to thank Maryline Talmant for her expert contribution to our research.

\section{References and links}

${ }^{1}$ S. R. Cummings and L. J. Melton, "Epidemiology and outcomes of osteoporotic fractures", Lancet 359, 1761-7 (2002).

${ }^{2}$ D. Marshall, O. Johnell, and H. Wedel, "Meta-analysis of how well measures of bone mineral density predict occurence of osteoporotic fractures", BMJ 312, 1254-1259 (1996).

${ }^{3}$ R. M. Zebaze, A. Ghasem-Zadeh, A. Bohte, S. Iuliano-Burns, M. Mirams, R. I. Price, E. J. Mackie, and E. Seeman, "Intracortical remodelling and porosity in the distal radius and postmortem femurs of women: a cross-sectional study", Lancet 375, 1729-36 (2010).

${ }^{4}$ G. Holzer, G. von Skrbensky, L. A. Holzer, and W. Pichl, "Hip fractures and the contribution of cortical versus trabecular bone to femoral neck strength", J. Bone Miner. Res. 24, 468-474 (2009).

${ }^{5}$ J. Grondin, Q. Grimal, K. Engelke, and P. Laugier, "Potential of first arriving signal to assess cortical bone geometry at the hip with QUS: a model based study", Ultrasound Med. Biol. 36, 656-66 (2010). 
${ }^{6}$ C. Prada and M. Fink, "Separation of interfering acoustic scattered signals using the invariants of the time-reversal operator. Application to Lamb waves characterization", J. Acoust. Soc. Am. 104, 801-807 (1998).

${ }^{7}$ M. Granke, Q. Grimal, A. Saïed, P. Nauleau, F. Peyrin, and P. Laugier, "Change in porosity is the major determinant of the variation of cortical bone elasticity at the millimeter scale in aged women", Bone 49, 1020-1026 (2011).

${ }^{8}$ S. A. Goss, R. L. Johnston, and F. Dunn, "Compilation of empirical ultrasonic properties of mammalian tissues. II", J Acoust Soc Am 68, 93-108 (1980).

${ }^{9}$ J. G. Minonzio, J. Foiret, M. Talmant, and P. Laugier, "Impact of attenuation on guided mode wavenumber measurement in axial transmission on bone mimicking plates", J. Acoust. Soc. Am. 130, 3574-3582 (2011).

${ }^{10}$ H. Blain, P. Chavassieux, N. Portero-Muzy, F. Bonnel, F. Canovas, M. Chammas, P. Maury, and P. D. Delmas, "Cortical and trabecular bone distribution in the femoral neck in osteoporosis and osteoarthritis", Bone 43, 862-8 (2008).

${ }^{11}$ G. V. Frisk and H. Überall, "Creeping waves and lateral waves in acoustic scattering by large elastic cylinders", J Acoust Soc Am 59, 46-54 (1976).

${ }^{12}$ X. L. Bao, H. Franklin, P. K. Raju, and H. Uberall, "The splitting of dispersion curves for plates fluid-loaded on both sides", J. Acoust. Soc. Am. 102, 1246-1248 (1997).

${ }^{13}$ E. Bossy, P. Laugier, F. Peyrin, and F. Padilla, "Attenuation in trabecular bone: a comparison between numerical simulation and experimental results in human femur", J. Acoust. Soc. Am. 122, 2469-75 (2007). 
Nauleau, JASA-EL

\section{Captions}

Figure 1: A hollow tube of diameter D immersed in water is excited with an array of transducers. Circumferential wave of velocity $c_{\phi}$ is generated in A, defined by $\theta$. This wave propagates in the shell and radiates in the external medium. The radiated wave is recorded by the array; the signal appears to come from a virtual source B'. Reciprocally, a circumferential wave is generated in B, and the radiated wave is seen coming from A' for the array.

Figure 2: Echoes of the tube received by each of the 128 transducers after an excitation. Two components are distinguished by a vertical blank line: a first echo associated with the specular echo of the tube and a second one corresponding to the circumferential waves. Thin horizontal blank lines correspond to faulty transducers.

Figure 3: Experimental dispersion curves of the bone-mimicking tube (circles) are superimposed with the curves obtained by analytical simulation of the experiment (crosses) and the theoretical curves (points) for a semi-immersed plate in the (frequency $f$, wavenumber $k$ ) plane. The dashed line represents negative values of wave number, associated with the backward mode $S_{2 b}$. 


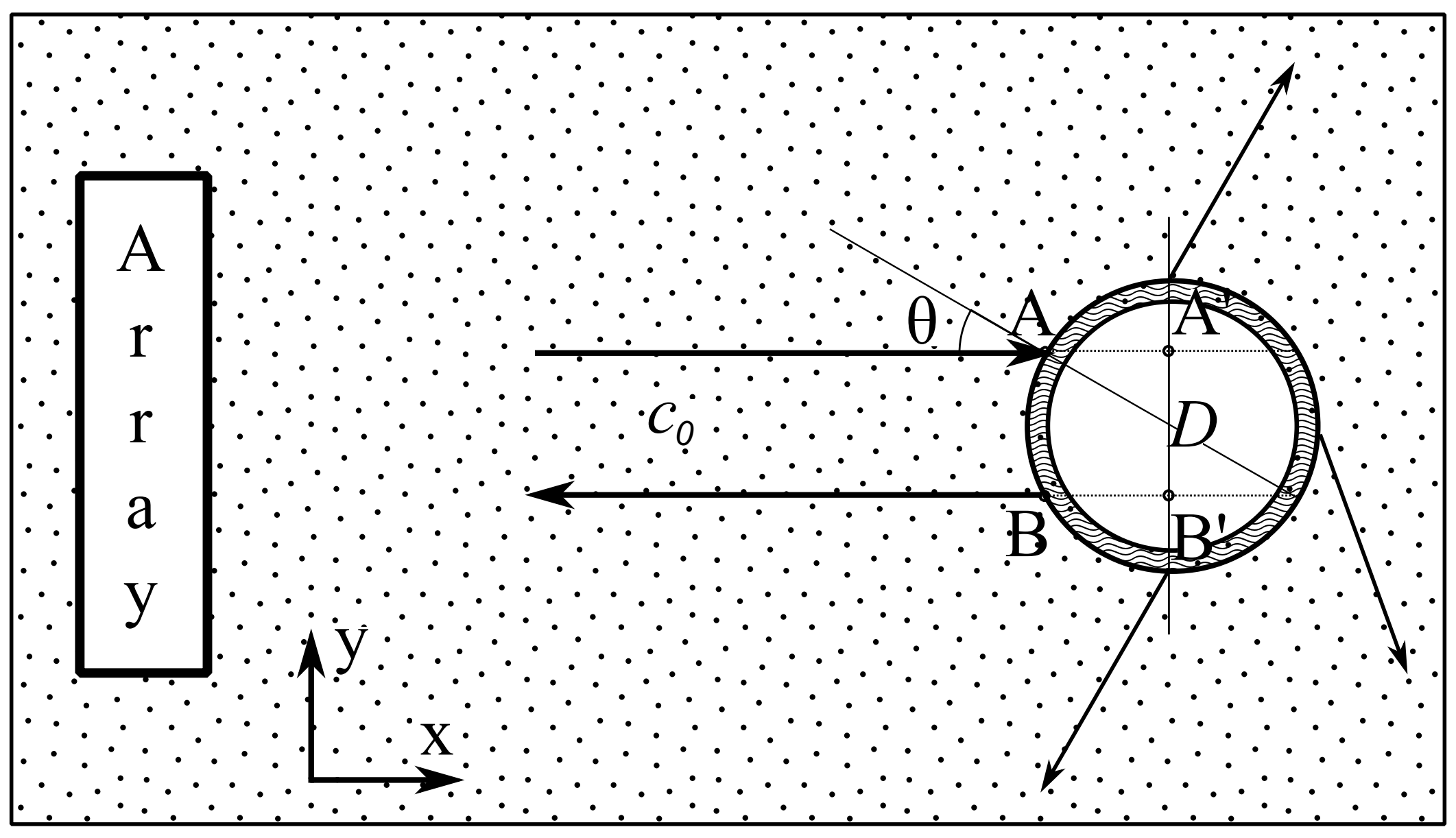



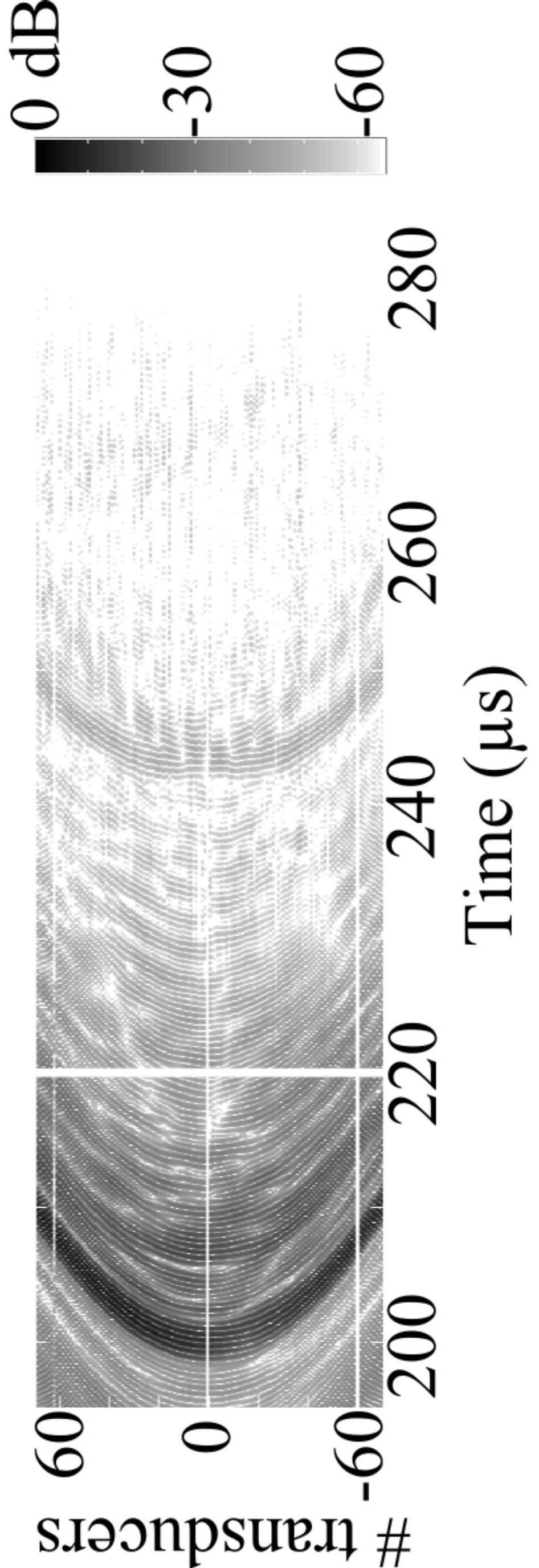


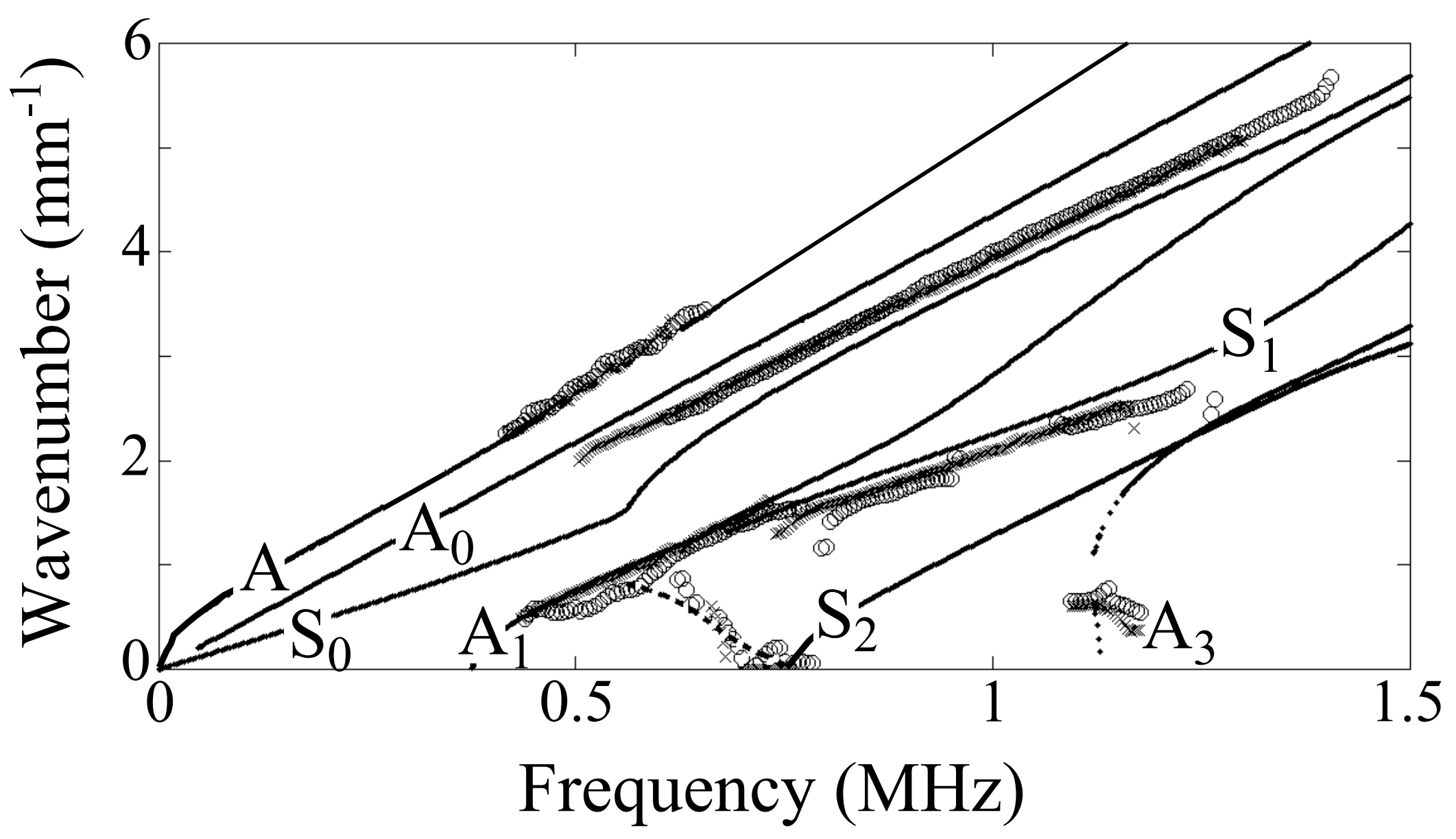

